\title{
ASCA OBSERVATIONS OF BLAZARS
}

\author{
T. TAKAHASHI \\ Institute of Space and Astronautical Science \\ 3-1-1 Yoshinodai, Sagamihara, Kanagawa, Japan \\ H. KUBO \\ The Institute of Physical and Chemical Research, 2-1 Hirosawa, Wako, Saitama, 351- \\ 01, Japan
}

AND

G. MADEJSKI

Laboratory for High Energy Astrophysics, Code 662, NASA GSFC, MD 20771, USA

\section{Introduction}

During the last years it has become evident that blazar class of AGN emit a lot of energy in the gamma-ray regime. It is generally thought that the non-thermal emission from blazars, observed from radio to $\mathrm{GeV} / \mathrm{TeV} \gamma$-rays, is radiation of very energetic particles via both synchrotron and Compton processes. This underlines the importance of high-energy emission for models of the blazar class of active galactic nuclei. Most of the gamma-bright AGN are variable, and flares with time scales as short as a day have been observed. This suggests that the site of gamma-ray emission is very compact and situated in a fluid which moves relativistically at a small angle towards the observer. It is generally assumed that the emission originates from the jet of these objects.

The overall spectra of blazars have two pronounced components in $\nu-F(\nu)$ representation (giving the emitted power per decade of energy): one peaking at low energies (LE), $10^{13}-10^{17} \mathrm{~Hz}$, and another peaking at high energies (HE), in the $\gamma$-rays. For the blazars that are hosted in quasars (QHBs), and for BL Lac objects discovered via radio-selection techniques (the so-called "Lowenergy peaked BL Lacs," or LBLs), the LE component peaks in the infrared. For the the majority of BL Lac objects - those found as a result of their X-ray emission - it peaks in the ultraviolet or even in the soft X-rays, and thus the name "High-energy peaked BL Lacs" (e.g., (Sambruna, Maraschi \& Urry 1996)). The local power-law shape, the smooth connection of the entire radio - to - UV (and, for the HBLs, soft X-ray) continuum, as well as the relatively high level of polarization observed from radio to the UV, implies that the emission from the LE component is most likely produced via the synchrotron process of relativistic particles radiating in magnetic field.

The HE component, on the other hand, peaks in the $\gamma$-ray band, in the MeV - to - GeV range, and, in the case of a few HBLs, it extends to the TeV range; it is believed to be produced via Comptonization by the same particles that radiate the LE component. The source of the "seed" photons, can either be the synchrotron radiation, internal to the jet - as in the Synchrotron-SelfCompton (SSC) models (e.g., Ghisellini \& Maraschi 1989). Alternatively, these can be external to the jet, as in the External Radiation Compton (ERC) models: either the UV accretion disk photons (Dermer, Schlickheiser, \& Mastichiadis 1992), or these UV photons reprocessed by the emission line clouds and/or intercloud medium (Sikora, Begelman \& Rees 1994; Blandford \& Levinson 1995), or else, IR radiation ambient to the host galaxy (Sikora, Begelman \& Rees 1994).

To study the multiband spectrum is very important to obtain the information of the physical conditions in the jet where the high-energy radiation is emitted. Importantly, the relationship of the variability patterns from one band to another provide additional information as to the relationship of the physical processes responsible for the emission in these bands. Since the launch 
of ASCA, extensive multiband campaigns of blazars have been carried out (Madejski et al. 1996; Takahashi et al. 1996a; Takahashi et al. 1997; Urry et al. 1997; Kubo et al. 1997). In this paper, we make a brief review of ASCA observations of blazars and their multiband analysis.

\section{ASCA Observations}

With satellite for X-ray astronomy, ASCA, we observed 18 blazars, of which 10 were also observed contemporaneously with the EGRET instrument onboard Compton Gamma-ray Observatory as parts of multi-wavelength campaigns. The observations show a clear difference in the spectra and variability between HBLs (H0323+022, H0414+009, PKS0548-322m MKn421, Mkn501, PKS2155-304), LBLs (AO0235+164, 0716+714, PKS0735+178, OJ287), and QHBs (PKS0208-512, NRAO140, PKS0528+134, 3C273, 3C279, 4C38.41, CTA102). The ASCA X-ray spectra of HBLs are the softest, with the power law energy index $\alpha \sim 1-2$, and they form the highest observable energy tail of the low energy (LE, synchrotron) component. The X-ray spectra of the QHBs are the hardest $(\alpha \sim 0.6)$ and are consistent with the lowest observable energy end of the high energy (HE, Compton) component. For LBLs, the X-ray spectra are intermediate (Kubo et al. 1997).

\section{Dynamics: the case of BL Lac object Mkn 421}

Among the GeV-emitting blazars, the BL Lac object Mkn 421 is unique as the first - and so far, the brightest member where the $\gamma$-ray emission extends up to the TeV energies at a level allowing detailed spectral and variability studies in the broadest range of wavebands. Mkn 421 is one of the best known and studied BL Lacs; it shows optical polarization, flat radio spectrum and significant time variability, characteristics of the blazar class. The simple continuum spectra of Mkn 421 from the radio to the $\mathrm{X}$-ray bands obtained previously imply that the $\mathrm{X}$-ray emission is due to the distribution of charged particles radiating via the synchrotron process (see, e.g., George et al. 1988), while the $\mathrm{GeV}$ and $\mathrm{TeV}$ emission is likely to be due to the Compton process from the same particles. The $\mathrm{X}$-ray emission and a comparison of it with that in the $\mathrm{GeV} / \mathrm{TeV}$ bands is thus crucial to study the emission mechanism of $\mathrm{GeV} / \mathrm{TeV}$ photons, and specifically, the distribution of the relativistic electrons.

The multi-frequency observation of Mkn 421 from the radio to $\mathrm{TeV} \gamma$-ray bands in 1994 resulted in the detection of a simultaneous $\mathrm{keV} / \mathrm{TeV}$ flare by the ASCA and Whipple Observatory (Macomb et al. 1995, Takahashi et al. 1996a). Notably, the GeV $\gamma$-ray flux observed by EGRET, as well as the radio and UV fluxes showed less variability than $\mathrm{keV}$ or $\mathrm{TeV}$ bands (Macomb et al. 1995). The ASCA observation lagged the onset of the TeV flare by one day but recorded a high level of $2-10$ $\mathrm{keV} \mathrm{X}$-ray flux peaking at $3.7 \times 10^{-10} \mathrm{ergs} \mathrm{cm}^{-2} \mathrm{~s}^{-1}$, a 10 -fold increase over the quiescent level. The 1995 multiwavelength campaign revealed another coincident $\mathrm{keV} / \mathrm{TeV}$ flare (Takahashi et al. 1996b; Buckley et al. 1996). Although the variations of relative amplitude are different, the optical and infrared energy band also showed correlation during the flares (Fig. 1). During two weeks of observations in 1995, the $\mathrm{X}$-ray light curve showed a general decline with a time scale of a week, with intra-day variations during most of the individual observations. The evolution of the $\mathrm{X}$-ray spectrum was clearly observed in both observations, as it became steeper in the declining phase and harder in the brightening phase. Hard $\mathrm{X}$-rays showed larger amplitude variations than the soft $\mathrm{X}$-rays below $2 \mathrm{keV}$.

Importantly, both 1994 and 1995 ASCA data allowed us to quantify the X-ray spectral change, such that the soft X-ray photons lag the harder X-rays by about $1 \mathrm{hr}$. The discovery of the soft $\mathrm{X}$-ray lag in the X-ray variability of Mkn 421 allows further constraints on the parameters of the emitting region. If this is due to cooling of electrons by synchrotron radiation, we can calculate the magnetic field (see, e.g., Tashiro et al. 1995; Takahashi et al. 1996a). Since the lag is the difference of the cooling time $t_{\text {sync }}$ of electrons radiating at different energies, we use the data shown in Fig. 6 to infer $t_{\text {sync }} \sim 6000 \mathrm{~s}$ at $1 \mathrm{keV}$. With this value, we calculate the magnetic field $B$ of $\sim 0.2\left(\delta_{5}\right)^{-1 / 3}$ Gauss (where $\delta_{5}=\delta / 5$ ). With this $B$, we calculate the $\gamma_{e l}$ of electrons responsible for $\mathrm{keV}$ emission to be $\sim 5 \times 10^{5} E^{1 / 2}$, where $E$ is the energy in units of keV. It should be noted that the similar result was obtained from PKS 2155-304 (Urry et al. 1997). In addition to the soft X-ray lag, the 
$\mathrm{X}$-rays led the ultraviolet by a few hours which might be explained by the difference of the emission region between $\mathrm{X}$-ray and EUV.

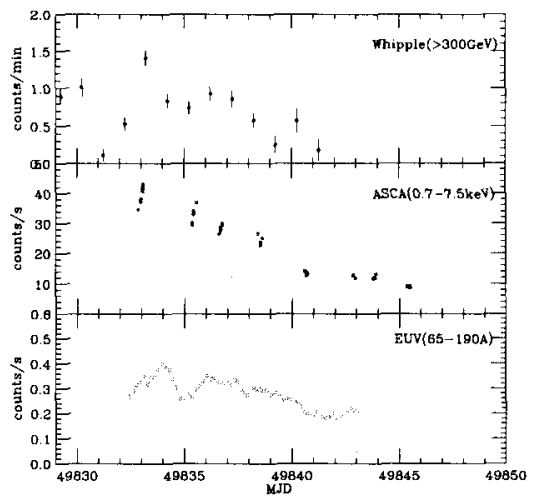

Fig. 1: Light curve obtained in 1995 campaign. Rapid variability is seen in all energy bands. Doubling times of order 1 day have been recorded in the X-ray (Takahashi et al. 1996b) an d TeV gamma-ray (Buckley et al. 1997) ranges. The EUV emission (Kartje et al. 1997) does follow the general decline after the gamma-ray peak in time-scale and amplitude. The X-ray light curve resolves intraday variations during most of the individual observations.

\section{Multiband analysis of blazars}

Two examples of blazar spectra plotted in $\nu F(\nu)$ are shown in Figures $2 \mathrm{a}$ and $2 \mathrm{~b}$. The overall spectra have at least two pronounced components: the LE and the HE. Fig. 2a shows the spectrum of Mkn 421, a fairly typical HBL; its X-ray spectrum is soft (steep). Fig. 2b shows PKS $0528+134$ (Collmar et al. 1997), a QHB; in contrast to Mkn 421, the X-ray spectrum of PKS $0528+134$ is harder than the UV spectrum, implying that the $\mathrm{X}$-rays are already not the synchrotron tail, but the onset of the second, HE component.

The multi-band analysis of the overall spectra of HBLs (Kubo et al. 1997) indicates that the luminosoty of the HE component is comparable to that of the LE component. Based on the Dopplerboosted Synchrotron-Self-Compton (SSC) model, the radiation losses due to synchrotron emission and to inverse Compton effect are in the same ratio as the magnetic field energy density and photon energy density (e.g. Rybicki and Lightman 1979). We find that the radiation process responsible for the HE peak for HBLs can be explained solely by SSC emission, with the Doppler factor $\delta$ consistent with the VLBI and/or $\gamma$-ray variability data. For many QHBs, on the other hand, the power in the HE to the LE components appears to be systematically larger for QHBs than for HBLs. In this case, the $\gamma$-rays cannot be solely due to the SSC mechanism unless $\delta$ is significantly in excess of that inferred from VLBI data. We consider an alternative scenario consistent with the measured values of $\delta$, where the SSC component is still present in QHBs and it dominates in the $\mathrm{X}$-ray band, but it is below the observed $\gamma$-ray spectrum. With an assumption that the peak of the SSC emission is on the extrapolation of the X-ray spectrum, we estimate the SSC luminosity. Once we obtain the SSC luminosity, the magnetic field strength can be calculated from the ratio of the luminosity of the SSC and the synchrotron components. Adopting $\delta$ of 10 , the magnetic fields are inferred to be $0.1 \sim 1$ Gauss. In particular, the value derived for Mkn421 is $\sim 0.1$ gauss, which is gratifyingly consistent with the value obtained from the argument of synchrotron cooling.

With these values of $B$, we have found that the Lorentz factor of electrons $\left(\gamma_{e l}\right)$ are calculated to be higher for HBLs - in which the LE component peaks at higher frequencies $\left(10^{16}-10^{17} \mathrm{~Hz}\right)$ than those for QHBs, where the peak is at lower frequencies $\left(10^{13}-10^{14} \mathrm{~Hz}\right)$. This picture is consistent with the fact that the $\mathrm{TeV}$ emission has been observed only from the HBLs, which turned out to have higher $\gamma_{e l}$. For QHBs, where a dense external radiation fields exist, the ERC emission most likely contributes as much or even more significantly than the SSC emission in $\gamma$-ray band (see, 
e.g., Sikora et al. 1997). Therefore, our analysis indicates that the difference of $\gamma_{e l}$ is related to the large photon density in QHBs as compared with that of HBLs; Compton upscattering of these photons may well provide the observed GeV flux.

\section{Summary}

Our results show that the non-thermal emission from blazars, observed from radio to $\mathrm{GeV} / \mathrm{TeV}$ $\gamma$-rays, is radiation of very energetic particles via both synchrotron and Compton processes. While the overall spectra of all blazars over this wide range of energies appear similar from one object to another, the $\mathrm{X}$-ray regime is an important band, where the emission due to both processes overlaps: it is either the high energy end of the synchrotron emission or the lowest observable energy end of the Comptonized spectrum. The ASCA data allow investigation of the details of the emission and estimation of the fundamental physical parameters, such as $B$ and $\gamma_{e l}$.

However, the details of the jet structure, and in particular, the question of the particle acceleration, still remain open. Correlating the interband variability can provide our best opportunity to understand high-energy emission from blazar jets. Previous multiwavelength campaigns revealed correlated variability but did not have sufficient sampling to permit firm conclusions about the conditions in the jet. We will undertake a 7.5 day campaign for Mkn 421 in 1998 with continuous coverage by ASCA, XTE, OSSE, EGRET and ground-based radio, optical and TeV observatories. This will provide a large improvement over previous sampling and permit quantitative studies of the conditions in the jet where the high-energy radiation is emitted.
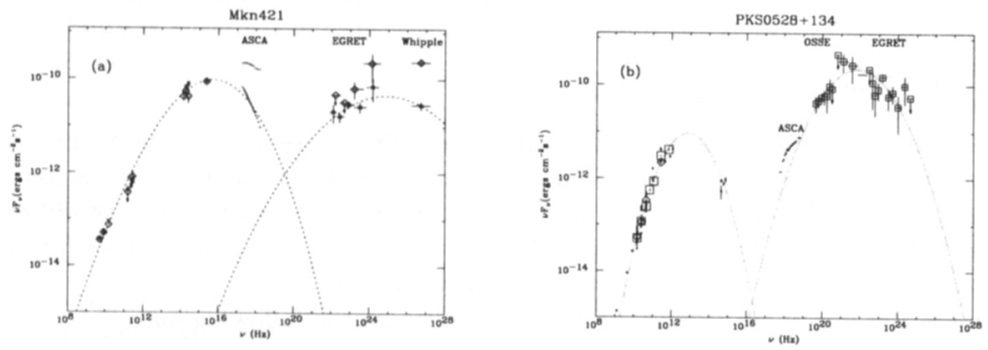

Fig. 2: The multiband spectrum obtained from the simultaneous observations of Mkn 421 and Quasar-Hosted Blazar PKS 0528+134. The dotted line shows the results of fit to a third-order polynomial function for low and high energy components.

\section{References}

Blandford, R. D., \& Levinson, A. 1995, ApJ, 441, 79

Buckley, J.H. et al., 1997, ApJ, 472, L9

Collmar, W., et al. 1997, in preparation

Dermer, C. D., Schlickeiser, R., \& Mastichiadis, A. 1992, A\&A, 256, L27

Ghisellini, G., \& Maraschi, L. 1989, ApJ, 340, 181

George, I., Warwick, R., \& Bromage, D. 1988, MNRAS, 232, 793

Kartje, J.F., et al., ApJ, 1997, 630;

Kubo, H., Takahashi, T., Madejski, G., Tashiro, M., Makino, F., Inoue, S., and Takahara, F, submitted

Macomb, D, et al. 1995, ApJ, 449, L99

Madejski, G. et al., 1996, $A p J, 459,156$

Sambruna, R. M., Maraschi, L., \& Urry, C. M. 1996, ApJ, 463, 444

Sikora, M., Begelman, M. C., \& Rees, M. J. 1994, ApJ, 421, 153

Sikora, M., Madejski, G., Moderski, R., \& Poutanen, J. 1997, ApJ, 484, 108

Takahashi, T., et al. 1996, ApJ, 470, L89

Takahashi, T., et al. 1996, Mem. Soc. Astron. Ital., 67, 533

Takahashi, T., et al. 1997, in Fourth Compton Symposium, in press

Tashiro, M. et al. 1995, PASJ, 47, 131

Urry, M. et al. 1997, ApJ, 487, 636 